\title{
Generation of microwave bursts with zebra pattern by nonlinear interaction of Bernstein modes ${ }^{\star}$
}

\begin{abstract}
A. A. Kuznetsov
Institute of Solar-Terrestrial Physics, Irkutsk, Russia

e-mail: a_kuzn@iszf.irk.ru

Received 17 January 2005 / Accepted 16 March 2005

Abstract. The processes of generation and nonlinear interaction of Bernstein modes are investigated. The physical conditions considered correspond here to the sources of microwave bursts with a zebra pattern. It is shown that the simultaneous generation of plasma waves corresponding to several cyclotron harmonics is possible under these conditions. Nonlinear coupling of plasma waves results in formation of a zebra pattern with a frequency separation between the adjacent stripes close to the electron cyclotron frequency. The emission is confined in the narrow angle interval near the transversal (relative to the magnetic field) propagation direction. Polarization of emission in this process corresponds to the extraordinary wave, and the polarization degree reaches $100 \%$.
\end{abstract}

Key words. radiation mechanisms: non-thermal - Sun: flares - Sun: radio radiation

\section{Introduction}

Solar radio bursts with a zebra type fine spectral structure, which consist of a number of parallel stripes in dynamic spectrum, have been investigated for many years. There are several approaches to interpretation of such structures, which were developed to apply to the bursts in the meter and decimeter ranges (Rosenberg 1972; Kuijpers 1975; Zheleznyakov \& Zlotnik 1975b; Chernov 1976, 1990; LaBelle et al. 2003). One of the reasons a more definite conclusion was not possible about the formation mechanism of zebra pattern was a lack of precise information about the spatial localization and size of the emission source.

Using the Siberian Solar Radiotelescope (SSRT) and the spectropolarimeters of the National Astronomical Observatories of China (NAOC), we at the first time managed to detect a zebra pattern in the microwave range (near 5.6 GHz) with high spatial resolution (down to $10^{\prime \prime}$ ). This event is described in detail in the article by Altyntsev et al. (2005). Figure 1 shows the dynamic spectrum and the emission temporal profiles of the burst with zebra pattern. This zebra pattern was observed both by the SSRT and by the NAOC spectropolarimeters in the emission with right circular polarization only, which suggests high (up to 100\%) circular polarization degree.

A comparison with the magnetogram shows that the emission source is situated above the region with the northern polarity magnetic field; thus the polarization sign

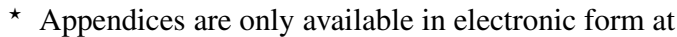
http://www. edpsciences.org corresponds to the extraordinary wave (X-mode) emission. This is quite an unexpected conclusion, so we investigated the possibility of polarization reversal. The observed polarization sign can differ from the polarization in the source if the emission crosses regions of quasi-transversal magnetic field or neutral current sheets during propagation from the source to the Earth (Zheleznyakov 1997). But reconstruction of the magnetic field structure from the magnetogram (Rudenko 2001) shows that in this event the observed zebra pattern emission does not cross the regions where polarization reversal can take place; therefore the high polarization degree, corresponding to the $\mathrm{X}$-mode, is formed in the emission source.

Direct measurements with the SSRT show that the emission source size is less than $10^{\prime \prime}$ and the sources of different zebra stripes spatially coincide. But the dynamic spectrum properties suggest even an stricter limitation on the source size. Indeed, the different zebra stripes have a very similar temporal evolution (including the simultaneous sudden onset of the frequency drift seen in Fig. 1), which requires a simultaneous change of source parameters, such as plasma density and magnetic field, in all parts of the source. The observed time delay of the frequency drift onset of different zebra stripes is not more than $50 \mathrm{~ms}$; multiplying this value by the Alfven velocity, that is, the velocity of the fastest disturbances affecting the magnetic field and plasma density in the magnetized plasma, results in a source size not more than one thousand $\mathrm{km}$ even for the upper estimations of the Alfven velocity.

Among the mechanisms that were proposed for interpreting zebra patterns, the compact source and mainly extraordinary wave can be explained by the process of nonlinear coupling of 


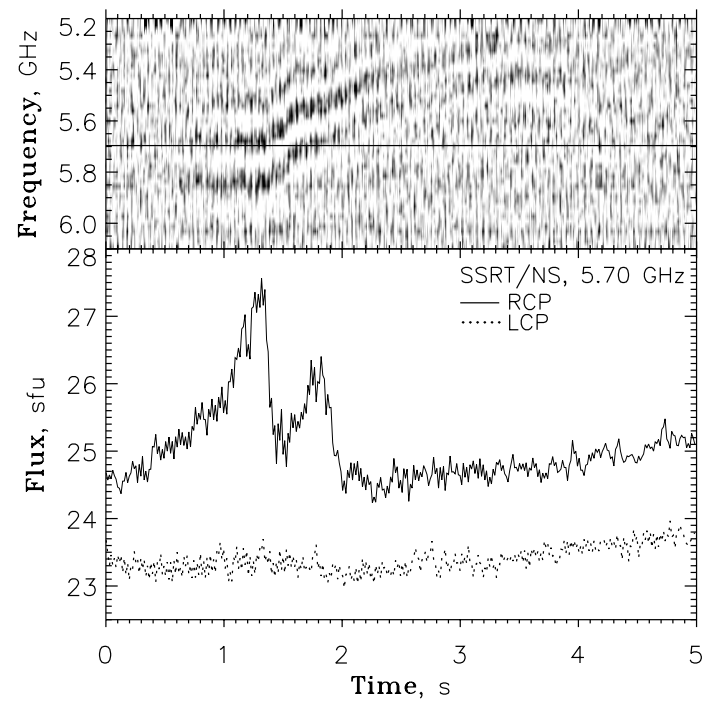

Fig. 1. Top: NAOC dynamic spectrum with zebra pattern, recorded on January 5, 2003. Bottom: the corresponding temporal profiles of emission with right (RCP) and left (LCP) circular polarization at $5.70 \mathrm{GHz}$, recorded by the SSRT.

Bernstein modes harmonics (Zheleznyakov \& Zlotnik 1975b; Mollwo \& Sauer 1977). The most complete investigation of this process has been done in the works of Willes \& Robinson (1996) and Willes (1999), but with application to relatively low cyclotron harmonics. At the same time, in the microwave zebra pattern (Fig. 1) the ratio of frequency interval between the adjacent zebra stripes to the emission frequency is $\Delta \omega / \omega \simeq 1 / 35$, which corresponds to the coalescence of Bernstein mode harmonics with numbers around 17-18.

The purpose of this work is to investigate the processes of generation and nonlinear interaction of Bernstein modes with application to conditions typical of the source of the microwave zebra pattern (Altyntsev et al. 2005).

\section{Generation of Bernstein modes}

\subsection{Dispersion equation}

The dispersion equation for the longitudinal plasma waves propagating perpendicular (or almost perpendicular) to the ambient magnetic field has the form (Zheleznyakov \& Zlotnik 1975a; Zheleznyakov 1997)

$\varepsilon_{\|}=1-2 \omega_{\mathrm{p}}^{2} \frac{\mathrm{e}^{-\lambda}}{\lambda} \sum_{r=1}^{\infty} \frac{r^{2} I_{r}(\lambda)}{\omega^{2}-r^{2} \omega_{\mathrm{B}}^{2}}=0$,

where $\omega, \omega_{\mathrm{B}}$, and $\omega_{\mathrm{p}}$ are the wave frequency, the electron cyclotron frequency, and the plasma (Langmuir) frequency, respectively; $\lambda=k_{\perp}^{2} v_{\mathrm{T}}^{2} / \omega_{\mathrm{B}}^{2}, k_{\perp} \simeq k$ is the transversal, relatively to the magnetic field, component of the wave vector $\boldsymbol{k}$ of the plasma waves; $v_{\mathrm{T}}=\sqrt{k_{\mathrm{B}} T_{0} / m_{\mathrm{e}}}$ is the mean thermal velocity of plasma electrons (with temperature $T_{0}$ ); and $I_{r}(\lambda)$ is a Bessel function of an imaginary argument. This equation has an infinite set of solutions with respect to the frequency for each value of wave vector. An example of dispersion curves is shown in Fig. 2. In the weakly anisotropic plasma $\left(\omega_{\mathrm{p}} \gg \omega_{\mathrm{B}}\right)$

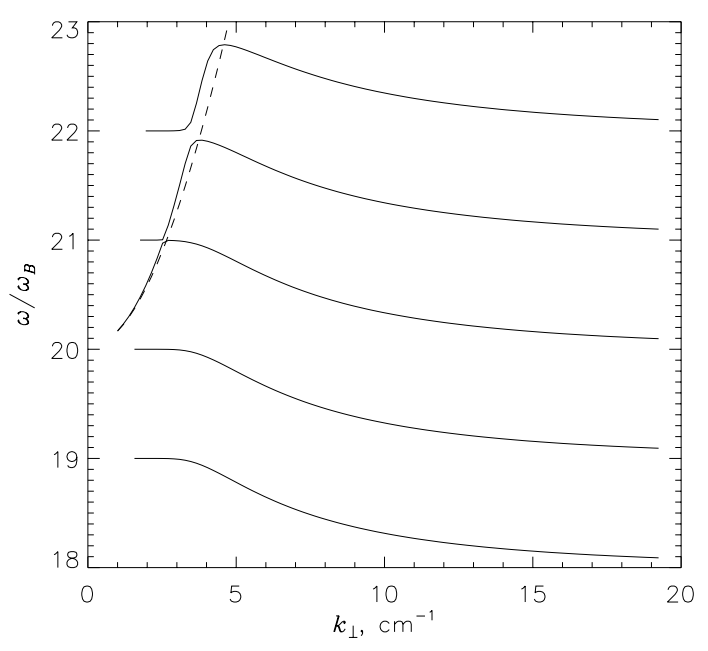

Fig. 2. Dispersion curves of longitudinal plasma waves. The parameters of plasma and magnetic field are given in Table 1. The dashed line shows the dispersion curve of upper-hybrid waves - the oscillation branch with normal dispersion law $\omega^{2} \simeq \omega_{\mathrm{p}}^{2}+\omega_{\mathrm{B}}^{2}+3 k_{\perp}^{2} v_{\mathrm{T}}^{2}$.

Table 1. The parameters of plasma, magnetic field, and accelerated particles used in the work.

\begin{tabular}{ll}
\hline \hline Plasma density: & $n_{0}=10^{11} \mathrm{~cm}^{-3}$ \\
Plasma temperature: & $T_{0}=10^{7} \mathrm{~K}$ \\
Plasma frequency: & $\omega_{\mathrm{p}}=2 \pi \times 2.85 \times 10^{9} \mathrm{~s}^{-1}$ \\
Magnetic field strength: & $B=50 \mathrm{G}$ \\
Cyclotron frequency: & $\omega_{\mathrm{B}}=2 \pi \times 1.42 \times 10^{8} \mathrm{~s}^{-1}$ \\
& $\omega_{\mathrm{p}} / \omega_{\mathrm{B}}=20$ \\
Accelerated particles distribution: & Dory-Guest-Harris \\
Accelerated particles temperature: & $T_{\mathrm{b}}=10^{8} \mathrm{~K}$ \\
Accelerated particles concentration: & $n_{\mathrm{b}}=n_{0} \times 10^{-2}$ \\
\hline
\end{tabular}

at $\omega<\omega_{\mathrm{p}}$ a set of almost equidistant curves with anomalous dispersion law exists; at $\omega>\omega_{\mathrm{p}}$ the regions with normal dispersion law appear. In this paper the integer value $s$, such that $s \omega_{\mathrm{B}}<\omega<(s+1) \omega_{\mathrm{B}}$, will be called the harmonic number of the Bernstein mode.

Equation (1) was obtained under the nonrelativistic approximation that is not valid within narrow frequency intervals $\Delta \omega_{r}$ below harmonics of cyclotron frequency $r \omega_{\mathrm{B}}, \Delta \omega_{r} / \omega \simeq v_{\mathrm{T}}^{2} / c^{2}$. It will be shown below that under these conditions the frequency of generated Bernstein modes differs sufficiently from the integer harmonics of cyclotron frequency, so the dispersion Eq. (1) is valid. In addition, the condition

$\left|\omega-r \omega_{\mathrm{B}}\right| \gg \sqrt{2} k_{\|} v_{\mathrm{T}}$

must be satisfied, where $k_{\|}$is the longitudinal component of the wave vector. A violation of this condition, which is a deviation from the quasi-transversal propagation, results in the heavy cyclotron damping of the plasma waves. 


\subsection{Growth rate}

The growth rate of longitudinal plasma waves is determined by the expression (Zheleznyakov \& Zlotnik 1975a; Zheleznyakov 1997)

$\gamma=-\frac{\operatorname{Im} \varepsilon_{\|}}{\left.\left[\partial \operatorname{Re} \varepsilon_{\|} / \partial \omega\right]\right|_{\operatorname{Re} \varepsilon_{\|}=0}}$,

where $\operatorname{Re} \varepsilon_{\|}$is determined by formula (1), and

$$
\begin{aligned}
\operatorname{Im} \varepsilon_{\|}= & -\pi m_{\mathrm{e}} \frac{\omega_{\mathrm{p}}^{2}}{k^{2}} \sum_{l=-\infty}^{\infty} \int\left(k_{\|} \frac{\partial f}{\partial p_{\|}}+\frac{l \omega_{\mathrm{B}}}{\Gamma v_{\perp}} \frac{\partial f}{\partial p_{\perp}}\right) \\
& \times J_{l}^{2}\left(\frac{\Gamma k_{\perp} v_{\perp}}{\omega_{\mathrm{B}}}\right) \delta\left[\Gamma\left(\omega-k_{\|} v_{\|}\right)-l \omega_{\mathrm{B}}\right] \mathrm{d}^{3} \boldsymbol{p} .
\end{aligned}
$$

Here $\boldsymbol{v}$ and $\boldsymbol{p}$ are the velocity and the impulse of electrons, $\Gamma=\left(1-v^{2} / c^{2}\right)^{-1 / 2}$, is the relativistic factor, while $f(\boldsymbol{p})$ is an electron distribution function that satisfies the normalization condition

$$
\int f(\boldsymbol{p}) \mathrm{d}^{3} \boldsymbol{p}=1,
$$

and $J_{l}(\zeta)$ is a Bessel function. Calculation of $\operatorname{Im} \varepsilon_{\|}$is reduced to calculating integrals along the resonance curves - arcs of ellipses $\Gamma\left(\omega-k_{\|} v_{\|}\right)-l \omega_{\mathrm{B}}=0$ (see Appendix A), and then the contributions of different cyclotron harmonics $l$ are added together. For those electrons with temperature about $10^{8} \mathrm{~K}$, it is enough to take 3-4 terms in sum (4) into account; with an increase in the typical energy of accelerated particles, the number of significant terms in the expression for $\operatorname{Im} \varepsilon_{\|}$increases, too.

\subsection{Distribution function of accelerated particles}

For the positive growth rate of plasma waves the presence of a region with positive slope on the distribution function $\left(\partial f / \partial p_{\perp}>0\right)$ is necessary. Let the distribution function be in the form

$f(\boldsymbol{p})=f_{0}(\boldsymbol{p})+f_{\mathrm{b}}(\boldsymbol{p})$,

where $f_{0}$ is the distribution function of background plasma (Maxwellian, with temperature $T_{0}$ and concentration $n_{0}$ ), and $f_{\mathrm{b}}$ is the nonequilibrium distribution function of accelerated particles. It is assumed that the dispersion properties of plasma are fully determined by the background component $f_{0}$.

Calculations show that the generation of high harmonics of Bernstein modes by the electron distribution of loss-cone type is ineffective. In this case the resonance curve passes through the region of positive slope on the distribution function (see Fig. 3) only for the waves that have essentially a nonzero longitudinal component of wave vector $k_{\|}$. For these waves condition (2) is not satisfied and Landau damping becomes important; in other words, other resonance curves make too strong a negative contribution to the sum over harmonics of cyclotron frequency.

The more effective source of Bernstein modes is the socalled ring beam with maximum at $\left\{v_{\|}=0 ; v_{\perp}=v_{\mathrm{b}}\right\}$ (Fig. 3).

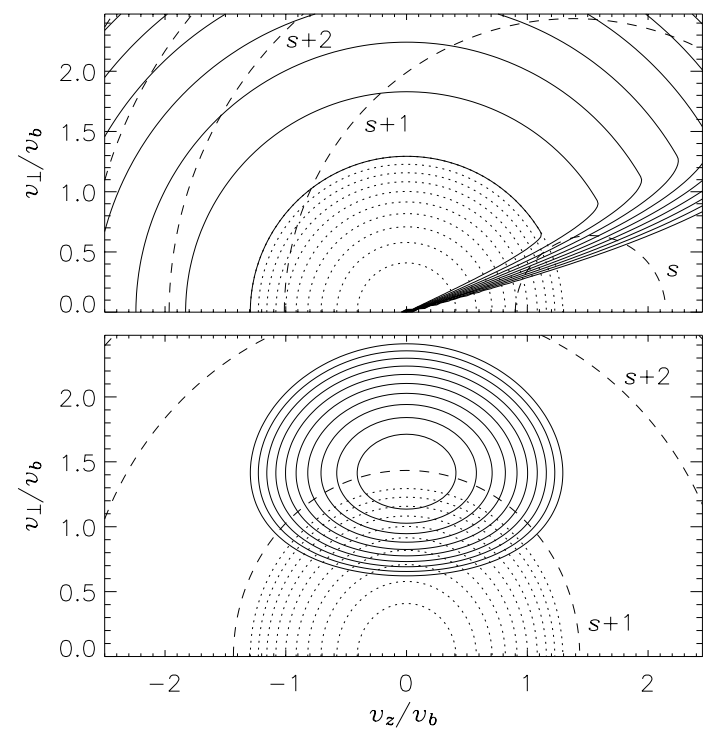

Fig. 3. Model distribution functions of accelerated electrons with losscone type (top) and ring beam type (bottom). The dashed lines show the resonance curves corresponding to the maximal growth rate for the Bernstein mode with frequency $s \omega_{\mathrm{B}}<\omega<(s+1) \omega_{\mathrm{B}}$ at different values of cyclotron harmonic number $l$. The dotted contours show the distribution of background plasma electrons.

This distribution can be simulated by the formula (Dory et al. 1965)

$f_{\mathrm{b}}\left(p_{\|}, p_{\perp}\right)=\frac{1}{\left(\sqrt{2 \pi} p_{\mathrm{b}}\right)^{3}} \frac{n_{\mathrm{b}}}{n_{0}} \frac{p_{\perp}^{2}}{2 p_{\mathrm{b}}^{2}} \exp \left(-\frac{p^{2}}{2 p_{\mathrm{b}}^{2}}\right)$,

where $p_{\mathrm{b}}=\sqrt{k_{\mathrm{B}} m_{\mathrm{e}} T_{\mathrm{b}}}$ is the typical impulse of accelerated particles; the temperature $T_{\mathrm{b}}$ and the concentration $n_{\mathrm{b}}$ of accelerated particles satisfy the relations $T_{\mathrm{b}} \gg T_{0}, n_{\mathrm{b}} \ll n_{0}$, $n_{\mathrm{b}} T_{\mathrm{b}} \ll n_{0} T_{0}$. Fulfilment of the last two inequalities allows us not to have to consider a contribution of the plasma hot component into the dispersion equation of plasma waves. In this case the waves propagating exactly perpendicular to the magnetic field $\left(k_{\|}=0\right)$ have maximal growth rate.

\subsection{Necessary conditions for generation}

Let us consider in detail the necessary conditions of longitudinal plasma waves generation with application to the waves propagating perpendicular to the magnetic field. In this case the resonance condition is reduced to the equation $\Gamma \omega-l \omega_{\mathrm{B}}=0$ or

$v=c \sqrt{1-\frac{\omega^{2}}{\left(l \omega_{\mathrm{B}}\right)^{2}}}$

where $l \geq s+1$. At this value of particle velocity, the distribution function must have a positive slope. The temperature values of accelerated electrons, which are favorable for generation of the harmonic $s=19$ at $l=s+1$, are shown in Fig. 4 . The relative values of the parameter $\partial \varepsilon_{\|} / \partial \omega$ that is contained in the expression for the growth rate (3), are shown in this figure, too. At either very small or very large values of wave vector $(\lambda \ll 1$ or $\lambda \gg 1)$ the parameter $\partial \varepsilon_{\|} / \partial \omega$ approaches infinity, 


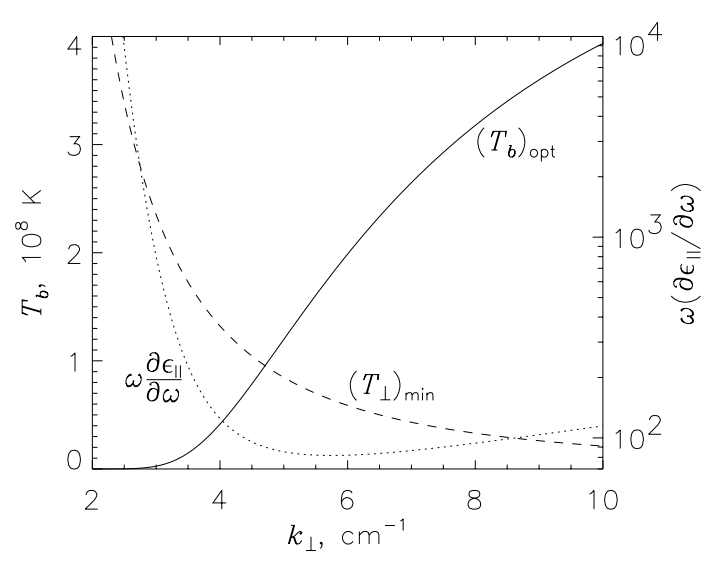

Fig. 4. Necessary conditions of Bernstein modes generation. The curve marked $\left(T_{\mathrm{b}}\right)_{\mathrm{opt}}$ shows the required typical temperature of accelerated electrons, obtained from the Eq. (8); the dashed curve $\left(T_{\perp}\right)_{\min }$ shows the required minimal value of the transversal component of electron temperature, derived from Eq. (9). The dependence of these values on the wave vector are calculated for the harmonic $s=19$, and the parameters of plasma and magnetic field given in Table 1 . The dotted line shows the corresponding values of the parameter $\omega\left(\partial \varepsilon_{\|} / \partial \omega\right)$.

which significantly decreases the efficiency of the generation of Bernstein modes, and the efficiency of their nonlinear transformation into radioemission, too. But under the conditions considered in this paper, the generation of plasma waves takes place within the region of wave vectors where the value $\partial \varepsilon_{\|} / \partial \omega$ is close to its minimum.

Another condition arises from the properties of the Bessel function that is contained in expression (4). The parameter $\Gamma k_{\perp} v_{\perp} / \omega_{\mathrm{B}}$ (the argument of Bessel function) in the resonance region must coincide with a maximum for function $J_{l}^{2}(\zeta)$, otherwise corresponding regions will not make a significant contribution to the growth rate. For sufficiently high harmonics $(s \simeq l>10)$ this requirement is reduced to

$$
\frac{k_{\perp} v_{\perp}}{\omega_{\mathrm{B}}} \gtrsim l \geq s+1
$$

This condition determines the lower boundary of the generation region with respect to wave vector $k_{\perp}$ or to the transversal component of the temperature of accelerated particles $T_{\perp}=m_{\mathrm{e}} v_{\perp}^{2} / k_{\mathrm{B}}$.

Figure 5 shows the typical values of wave vectors of the Bernstein modes that are generated by electrons with different energy. When analysing the generation of zebra pattern, the question is important: which modes of plasma waves will be predominantly generated under the given conditions? It is seen from Fig. 2 that for the formation of zebra pattern the generation of several harmonics of waves with anomalous dispersion law is necessary. At the same time, the upper-hybrid waves (the waves with normal dispersion law) have a much higher growth rate (Zheleznyakov \& Zlotnik 1975a). It then follows from Fig. 5 that at electron energy $T_{\perp}$ about $10^{8} \mathrm{~K}$ and background plasma temperature $T_{0}=10^{7} \mathrm{~K}$ the condition of generation (9) is not satisfied for the upper-hybrid waves. Therefore the energy of the unstable electron distribution will be distributed among the harmonics of plasma waves with an anomalous dispersion law.

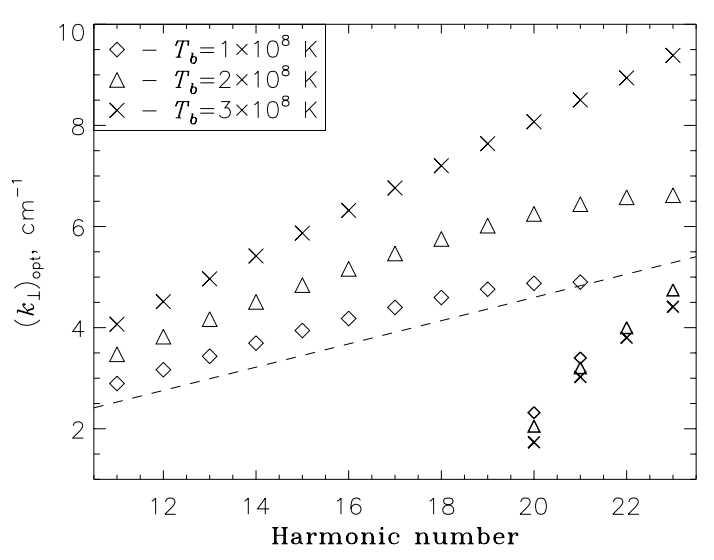

Fig. 5. Necessary conditions of Bernstein modes generation: the dependence of the typical wave vector on the harmonic number for the different values of the accelerated electrons temperature. The parameters of plasma and magnetic field are given in Table 1. The dashed line, corresponding to the minimal possible value of the wave vector (9), is drawn for $T_{\perp}=1 \times 10^{8} \mathrm{~K}$. In the right bottom corner of the figure the points are shown, corresponding to the branch of oscillations with normal dispersion law.

\subsection{Frequency and angular spectra}

In subsequent calculations in this paper we use the parameters of the accelerated electrons distribution listed in Table 1. The accelerated electron temperature is chosen to equal $10^{8} \mathrm{~K}$, because this value is high enough, in comparison with the background plasma temperature, to provide an effective plasma wave generation and, as shown above, ensures predominant generation of Bernstein modes. The Landau damping is taken into account by including the background plasma in the distribution function (6).

In Fig. 6 the maximal growth rates of plasma waves for different harmonics $s$ are shown. With an increase of harmonic number, the growth rate decreases; nevertheless the growth rates of different harmonics are comparable. The frequency interval between the waves of adjacent harmonics is close to the electron cyclotron frequency $\omega_{\mathrm{B}}$. Note in addition that the frequencies of plasma waves can significantly differ from the integer harmonics of cyclotron frequency $(s+1) \omega_{\mathrm{B}}$; the difference is about $\Delta \omega_{s} / \omega \simeq v_{\mathrm{e}}^{2} / c^{2}$, where $v_{\mathrm{e}} \gtrsim \sqrt{k_{\mathrm{B}} T_{\mathrm{b}} / m_{\mathrm{e}}}$ is the typical velocity of electrons generating those waves.

In Fig. 7 the dependence of the growth rate on the wave parameters is shown in detail; here $\theta$ is the angle between the wave vector and the magnetic field. The complicated shape of the dependence $\gamma\left(k_{\perp}\right)$ arises from the oscillating behaviour of function $J_{l}^{2}\left(\Gamma k_{\perp} v_{\perp} / \omega_{\mathrm{B}}\right)$ in the resonance region. Note in addition that the plasma waves are confined in the narrow angular interval, less than $0.4^{\circ}$, which agrees with condition (2). This peculiarity causes the narrow angular spectrum of the generated radioemission. 


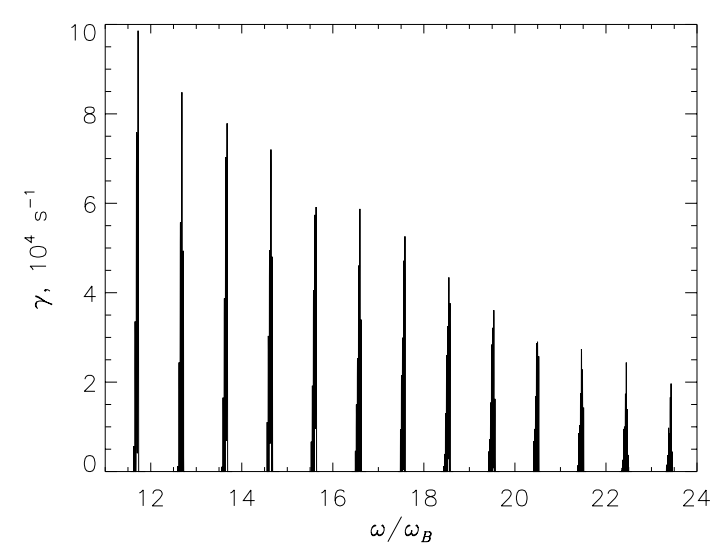

Fig. 6. Dependence of the growth rate of Bernstein modes on frequency for the transversal propagation. The parameters of plasma and magnetic field are given in Table 1 .

\section{Nonlinear interaction of Bernstein modes}

\subsection{Kinematic invariants and their consequences}

The process of merging two plasma waves with the parameters $\left(\omega^{\prime}, \boldsymbol{k}^{\prime}\right)$ and $\left(\omega^{\prime \prime}, \boldsymbol{k}^{\prime \prime}\right)$ into the electromagnetic wave with the parameters $(\omega, \boldsymbol{k})$ satisfies the kinematic conditions

$\omega=\omega^{\prime}+\omega^{\prime \prime}, \quad \boldsymbol{k}=\boldsymbol{k}^{\prime}+\boldsymbol{k}^{\prime \prime}$.

This results in the following consequences. First, the absolute values of wave vectors of plasma waves far exceed the absolute value of the wave vector of an electromagnetic wave. Therefore the wave vectors of interacting plasma waves must be almost oppositely directed and the condition

$\left|k_{\perp}^{\prime}-k_{\perp}^{\prime \prime}\right| \leq k \simeq \frac{\sqrt{3}}{2} \frac{\omega}{c}$

must be satisfied. This requirement can affect the frequency interval between the stripes in the zebra pattern; if only the plasma waves of the same harmonics can be involved in the interaction, then the interval equals $\Delta \omega \simeq 2 \omega_{\mathrm{B}}$. If the interaction of the waves of adjacent harmonics is possible, then $\Delta \omega \simeq \omega_{\mathrm{B}}$. It is seen in Fig. 5 that the absolute values of the wave vectors of generated waves increase with an increase in harmonic number, but the difference of the wave vectors of adjacent harmonics does not exceed the absolute value of the wave vector of an electromagnetic wave, that is, about $1 \mathrm{~cm}^{-1}$ under the considered conditions. Thus we can conclude that the frequency interval between the stripes of zebra pattern will equal the electron cyclotron frequency in the emission source.

Second, for the longitudinal (parallel to the magnetic field) components of the wave vectors of the interacting waves the relation $k_{\|} \leq k_{\| \max }^{\prime}+k_{\| \max }^{\prime \prime}$ is satisfied, where $k_{\| \max }^{\prime}$ and $k_{\| \max }^{\prime \prime}$ are the longitudinal components of wave vectors of Bernstein modes which correspond to the largest possible deviation from the transversal propagation. This results in the limitation of the angular spectrum width of the generated radio emission:

$\frac{\Delta \theta}{\Delta \theta^{\prime}} \simeq \frac{2 k^{\prime}}{k}$

obtained under the assumptions $\Delta \theta^{\prime} \simeq \Delta \theta^{\prime \prime} \ll 1, k^{\prime} \simeq k^{\prime \prime}$. Under the considered conditions $\left(\Delta \theta^{\prime}<0.4^{\circ}\right)$ one finds that the

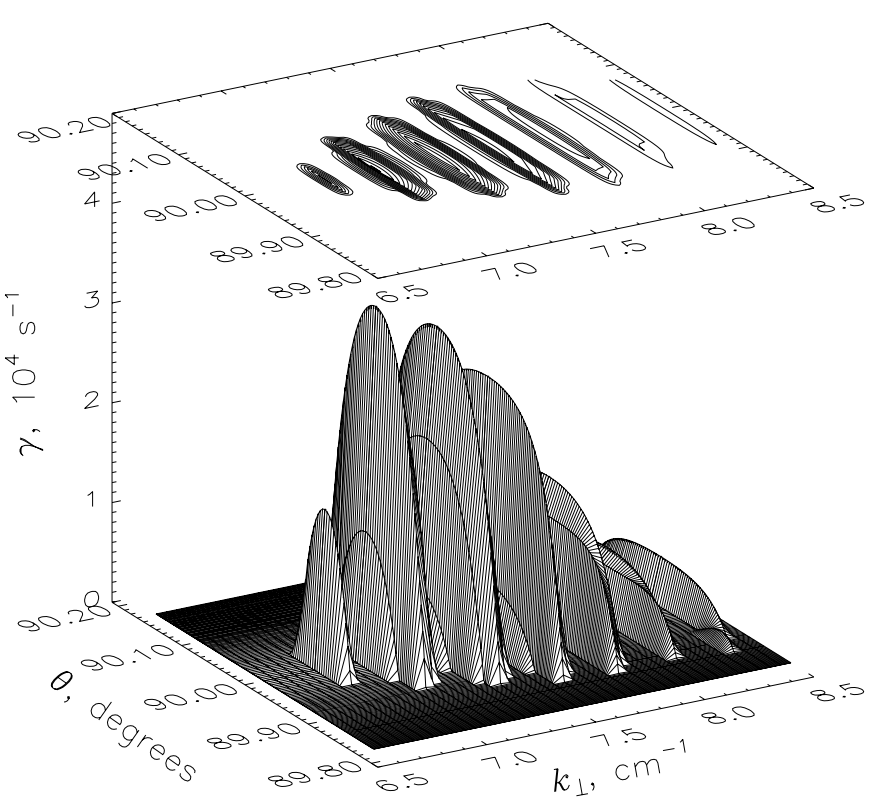

Fig. 7. The dependence of the growth rate of Bernstein modes on the wave vector and the propagation direction for the harmonic $s=19$.

width of angular spectrum of radioemission $\Delta \theta$ is about a few degrees. Thus the coupling of Bernstein modes results in generation of radioemission that is directed almost across the magnetic field.

\subsection{Radio emission intensity}

To calculate the intensity and polarization parameters of radio emission we will use the equation (Trakhtengerts 1970; Zlotnik 1981) describing the random-phase wave interaction:

$$
\begin{aligned}
& \frac{\mathrm{d} W_{\boldsymbol{k}}(\boldsymbol{k})}{\mathrm{d} t}=\int \Pi\left(\boldsymbol{k}, \boldsymbol{k}^{\prime}, \boldsymbol{k}^{\prime \prime}\right) W_{\boldsymbol{k}^{\prime}}^{\prime}\left(\boldsymbol{k}^{\prime}\right) W_{\boldsymbol{k}^{\prime \prime}}^{\prime \prime}\left(\boldsymbol{k}^{\prime \prime}\right) \\
& \times \delta\left(\omega-\omega^{\prime}-\omega^{\prime \prime}\right) \delta\left(\boldsymbol{k}-\boldsymbol{k}^{\prime}-\boldsymbol{k}^{\prime \prime}\right) \mathrm{d}^{3} \boldsymbol{k}^{\prime} \mathrm{d}^{3} \boldsymbol{k}^{\prime \prime},
\end{aligned}
$$

where $W_{k}, W_{\boldsymbol{k}^{\prime}}^{\prime}$, and $W_{\boldsymbol{k}^{\prime \prime}}^{\prime \prime}$ are the energy densities of the interacting waves in the space of the corresponding wave vectors. $\Pi\left(\boldsymbol{k}, \boldsymbol{k}^{\prime}, \boldsymbol{k}^{\prime \prime}\right)$ is the parameter describing the three-wave process probability:

$$
\begin{aligned}
& \Pi\left(\boldsymbol{k}, \boldsymbol{k}^{\prime}, \boldsymbol{k}^{\prime \prime}\right)=8(2 \pi)^{4} R(\boldsymbol{k}) R^{\prime}\left(\boldsymbol{k}^{\prime}\right) R^{\prime \prime}\left(\boldsymbol{k}^{\prime \prime}\right)|S|^{2}, \\
& S= {\left[\mathrm{S}_{i n m}\left(\boldsymbol{k}, \boldsymbol{k}^{\prime}, \boldsymbol{k}^{\prime \prime}\right)+\mathrm{S}_{i m n}\left(\boldsymbol{k}, \boldsymbol{k}^{\prime \prime}, \boldsymbol{k}^{\prime}\right)\right] } \\
& \times a_{i}^{*}(\boldsymbol{k}) a_{n}^{\prime}\left(\boldsymbol{k}^{\prime}\right) a_{m}^{\prime \prime}\left(\boldsymbol{k}^{\prime \prime}\right) .
\end{aligned}
$$

$\mathrm{S}_{i n m}$ is the nonlinear plasma conductivity tensor (the tensor that is used in this work is given in Appendix C); $\boldsymbol{a}, \boldsymbol{a}^{\prime}$, and $\boldsymbol{a}^{\prime \prime}$ are the unimodular polarization vectors (electric field vectors) of the corresponding waves; $R, R^{\prime}$, and $R^{\prime \prime}$ are the parameters that describe the ratio of the electric to total energy for the corresponding wave (these parameters together with the polarization vectors are given in Appendix B). For the interaction of plasma waves of the same type with the same distribution, the additional factor $1 / 2$ arises in expression (14). The terms describing the reverse process of decay of electromagnetic wave into two plasma waves are omitted in expression (13), as it is 
assumed that the energy density of electromagnetic waves is far less than the energy density of Bernstein modes, due to the rapid escape of the radio emission from the generation region.

The spectral intensity of the radioemission, observed at the Earth, under the optically thin source approximation is

$I_{f} \simeq \frac{2 \pi k^{2}}{v_{\mathrm{gr}}} \frac{V}{\mathcal{R}^{2}} \frac{\mathrm{d} W_{k}}{\mathrm{~d} t}$,

where $v_{\mathrm{gr}} \simeq c$ is the group velocity of the electromagnetic waves, $V$ is the characteristic volume of the emission source, and $\mathcal{R}$ is the distance between the Sun and the Earth.

\subsection{Polarization of radio emission}

We consider the case of weakly anisotropic plasma, and also it is assumed that the emission frequency significantly exceeds the extraordinary wave cutoff point. Under these conditions the dispersion properties of ordinary and extraordinary electromagnetic waves are similar, and the appearance of the high polarization degree is hard to expect. An exception is the case of quasi-transversal propagation, when the polarization of electromagnetic waves becomes linear; in the ordinary wave the electric field vector is parallel to the ambient magnetic field, and in the extraordinary wave the electric field vector is perpendicular to the ambient magnetic field.

Let us consider the situation when all of the interacting waves, including both the Bernstein modes and the electromagnetic wave, propagate exactly across the magnetic field. Bernstein modes are almost longitudinal waves; therefore in the coordinate system with axis $z$ parallel to the ambient magnetic field $\boldsymbol{B}$ (Fig. B.1), the components $a_{z}^{\prime}$ and $a_{z}^{\prime \prime}$ of the polarization vectors of the interacting plasma waves equal zero. The polarization vector of the ordinary electromagnetic wave propagating perpendicular to the magnetic field has the form $\boldsymbol{a}_{\mathrm{O}}=\left\{0,0, a_{z}\right\},\left|a_{z}\right|=1$; for the extraordinary wave one obtains $\boldsymbol{a}_{\mathrm{X}}=\left\{a_{x}, a_{y}, 0\right\},\left|a_{x}\right|^{2}+\left|a_{y}\right|^{2}=1$. In the considered coordinate system the components of the plasma nonlinear conductivity tensor $\mathrm{S}_{z x x}=\mathrm{S}_{z x y}=\mathrm{S}_{z y x}=\mathrm{S}_{z y y}=0$, as the perpendicular to the magnetic field components of the electric field do not affect the electron motion in the direction parallel to the magnetic field. It is easy to see that under these conditions the convolution $S$ (15) and, consequently, the three-wave process probability for the ordinary wave equal zero; on the contrary, for the extraordinary wave the three-wave process probability has nonzero values. Thus for the quasi-transversal propagation the generation conditions for the extraordinary wave are much more favorable than for the ordinary one, and the degree of polarization can reach $100 \%$.

In the weakly anisotropic plasma the quasi-transversal approximation for the electromagnetic waves propagation is valid only within a very narrow angle interval (Zheleznyakov 1997). Therefore for the plasma waves with a relatively broad angular distribution the effect above described can be neglected (Stepanov et al. 1999). On the contrary, it has been shown above that for the interaction of Bernstein modes the kinematic conditions of coupling (10) are satisfied only for the angular interval of emission propagation where the quasi-transversal approximation is valid.

\subsection{Results of numerical simulation}

For more accurate estimation of the radio emission parameters we use expression (C.1) for the plasma nonlinear conductivity tensor (Trakhtengerts 1970). This expression was obtained under the quasi-hydrodynamic approximation, and it is applicable for the weakly-damped oscillations of various types in the anisotropic medium.

The energy density distribution of Bernstein modes in the space of wave vectors is described by the following model:

$W_{\boldsymbol{k}^{\prime}}^{\prime}= \begin{cases}\frac{W^{\prime}}{\Delta^{3} \boldsymbol{k}^{\prime}}, & \text { when } k_{\min }^{\prime} \leq k^{\prime} \leq k_{\max }^{\prime} \text { and } \\ & \left|\frac{\pi}{2}-\theta^{\prime}\right| \leq \frac{\Delta \theta^{\prime}}{2}, \\ 0, & \text { in the other cases, }\end{cases}$

where $\Delta^{3} \boldsymbol{k}^{\prime} \simeq 2 \pi \Delta \theta^{\prime}\left[\left(k_{\max }^{\prime}\right)^{3}-\left(k_{\min }^{\prime}\right)^{3}\right] / 3$ is the phase volume occupied by the plasma waves, and $W^{\prime}$ is the total energy density. The boundary values of $k_{\min }^{\prime}$ and $k_{\max }^{\prime}$ for each harmonic are determined using the calculated values of the growth rate (see Figs. 6 and 7); namely, the requirement is used that at boundary values of the wave vector, the growth rate equals $1 / \mathrm{e}$ of its maximal value. Under the considered conditions for the harmonics $11 \leq s \leq 23$ the boundaries of the Bernstein modes generation region can be approximated with good precision by the following linear dependencies:

$k_{\min }^{\prime} \simeq 0.389 \mathrm{~s}-0.458 \mathrm{~cm}^{-1}$,

$k_{\max }^{\prime} \simeq 0.433 s-0.360 \mathrm{~cm}^{-1}$.

The angular spectrum width for all harmonics is assumed to equal $\Delta \theta^{\prime} / 2=0.2^{\circ}$.

The numerical simulation results for the process of Bernstein modes nonlinear interaction are shown in Figs. 8-11. In Fig. 8 the maximal transformation coefficient of plasma waves energy into electromagnetic waves $\left(\mathrm{d} W_{k} / \mathrm{d} t\right) /\left(W^{\prime} W^{\prime \prime}\right)$ for the different harmonics is shown. The energy transformation efficiency increases with an increase of the harmonic number. Note in addition that the efficiency of emission generation due to coalescence of the adjacent harmonics of Bernstein modes $\left(s^{\prime \prime}=s^{\prime}+1\right)$ is comparable with the efficiency of emission generation in the case of coalescence of waves with the same harmonic number $\left(s^{\prime \prime}=s^{\prime}\right)$. Thus the frequency interval between the adjacent stripes in the zebra pattern will be close to the electron cyclotron frequency. The simulations show that the efficiency of emission generation decreases sharply for the case $s^{\prime \prime}=s^{\prime}+2$.

Examples of calculation of the intensity observed at the Earth of radio emission of different modes, where the possible damping and scattering during propagation are not taken into account, are shown in Figs. 9 and 10. Based on observations, the size of emission source is assumed to be about one thousand $\mathrm{km}$; the required energy density of Bernstein modes in this case is $10^{-3}-10^{-2} \mathrm{erg} / \mathrm{cm}^{3}$. These values are rather small in comparison to the energy density of the background plasma or of the accelerated electrons: $W^{\prime} /\left(n_{0} k_{\mathrm{B}} T_{0}\right) \simeq 10^{-5}-10^{-4}$; $W^{\prime} /\left(n_{\mathrm{b}} k_{\mathrm{B}} T_{\mathrm{b}}\right) \simeq 10^{-4}-10^{-3}$.

It has been already noted that emission in the generation region is confined within the narrow angle interval, and the 


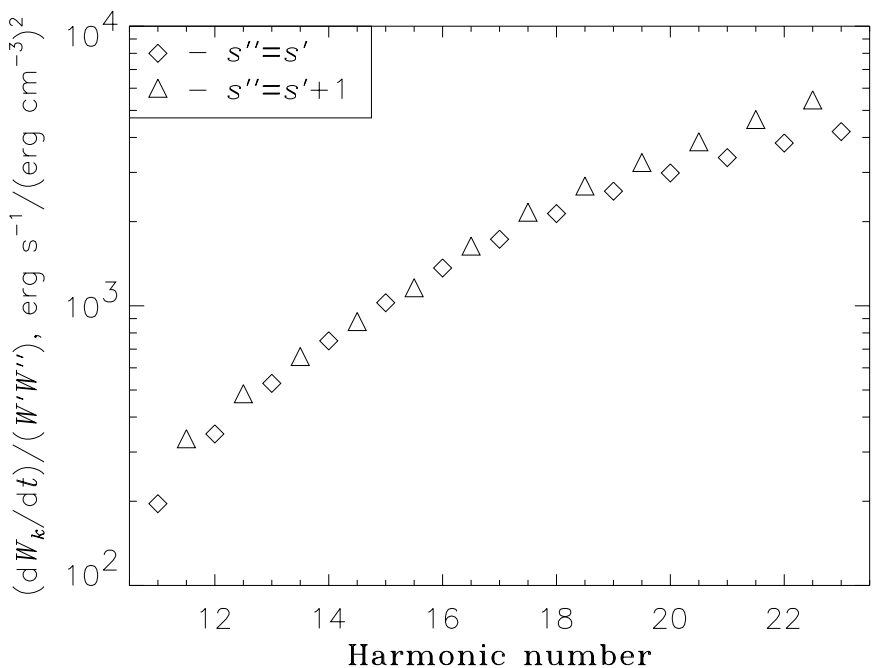

Fig. 8. The transformation coefficient of the energy of Bernstein modes into the electromagnetic waves, for the different numbers of merging harmonics. The maximal transformation coefficient is shown, which corresponds to the transversal propagation of emission and extraordinary wave. The case $s^{\prime \prime}=s^{\prime}$ corresponds to the coalescence of waves of the same type; $s^{\prime \prime}=s^{\prime}+1$ corresponds to the coalescence of waves of adjacent harmonics.

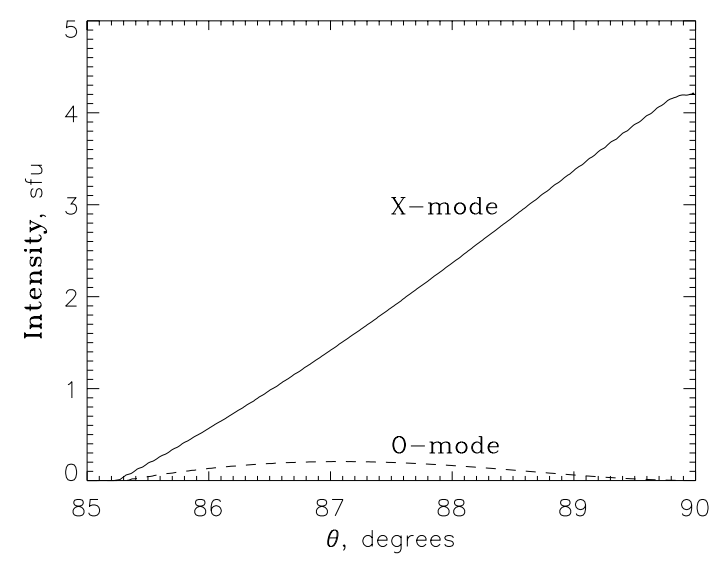

Fig. 9. The angular dependence of the spectral intensity of different modes of emission (at the distance of the Earth orbit radius), that is generated due to coalescence of Bernstein modes with the harmonic numbers $s^{\prime}=s^{\prime \prime}=11$. Plasma waves energy density $W^{\prime}=0.01 \mathrm{erg} / \mathrm{cm}^{3}$, emission source size $V=(1000 \mathrm{~km})^{3}$.

intensity maximum corresponds to the transversal to the magnetic field propagation direction. It is seen from the figures that the coalescence of Bernstein modes results in generation of a mainly extraordinary wave, and the polarization degree equals $100 \%$ for the transversal propagation direction. With deviation from the transversal propagation the polarization degree decreases somewhat; nevertheless it remains high for most of the angular interval of generation.

The frequency spectrum of emission (Fig. 11) that is generated due to coalescence of Bernstein modes is rather narrowband: $\Delta \omega_{\text {strip }} / \omega \simeq 0.16 \%$ at the level $1 / \mathrm{e}$ of the maximal intensity. The observed spectral width of the microwave zebra pattern stripes is somewhat larger, possibly caused by

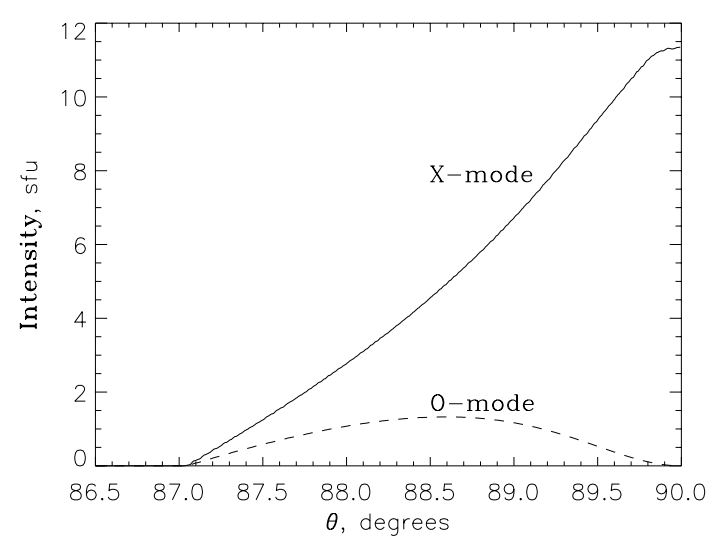

Fig. 10. The same as in Fig. 9, but for the harmonics $s^{\prime}=s^{\prime \prime}=19$; $W^{\prime}=0.002 \mathrm{erg} / \mathrm{cm}^{3}$.

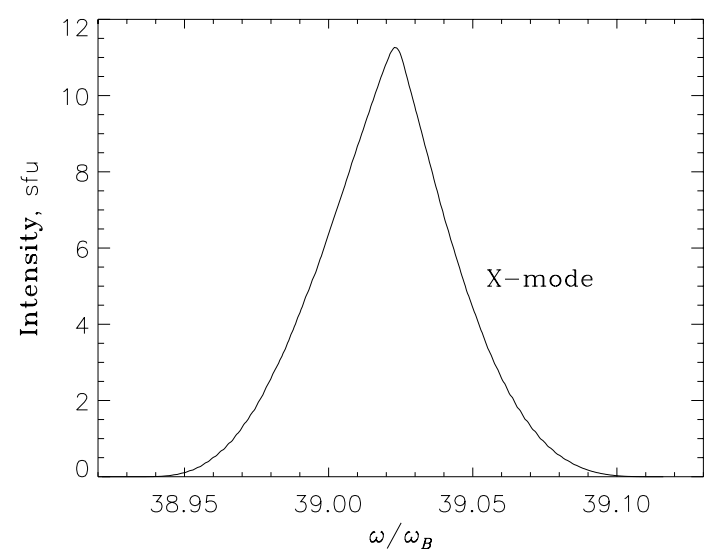

Fig. 11. Frequency dependence of the radio emission intensity for the same parameters as in Fig. 10. The maximal intensity is shown, which corresponds to the transversal emission propagation and extraordinary wave.

inhomogeneities of the magnetic field and plasma in the emission source.

\section{Discussion}

The investigation carried out shows that the simultaneous generation of a number of Bernstein mode harmonics in the active regions of solar corona is quite possible, but only with fulfilment of some requirements. In particular, the unstable electron distribution must have a ring beam shape in order to provide the possibility to generate plasma waves with a transversal propagation direction. Another important parameter is the ratio of energies of the accelerated electrons to the electrons of background plasma $T_{\mathrm{b}} / T_{0}$. Simulations show that when the background plasma temperature is $T_{0}=10^{7} \mathrm{~K}$, generation of the high harmonics of Bernstein modes requires the typical temperature of accelerated electrons in the distribution (7) to be in the interval $T_{\mathrm{b}} \simeq(0.9-3.5) \times 10^{8} \mathrm{~K}$; the electrons with lower energy will not be able to amplify the plasma waves due to the Landau damping, while the electrons with higher energy will amplify mainly upper-hybrid waves.

The transformation coefficient of the energy of Bernstein modes into electromagnetic waves increases with an increase in 
harmonic number (Fig. 8). This effect can limit the frequency of the observed stripes of zebra pattern from below; besides, the conditions of escape of the emission from plasma set the limitation $\omega>\omega_{\mathrm{p}}$. On the other hand, the generation efficiency of Bernstein modes decreases with an increase in harmonic number (Fig. 6). This effect limits the frequency of zebra pattern stripes from above. The estimations show that even for the saturated generation regime, when the plasma wave energy density is proportional to the growth rate, the influence of the last effect is more essential; so one can expect generation of emission at the intermediate frequencies: slightly above the plasma frequency $\omega_{\mathrm{p}}$, but considerably below the double plasma frequency $2 \omega_{\mathrm{p}}$. In the event of January 5, 2003 the interval favorable for generation contains 2-3 harmonics of Bernstein modes which correspond to 3-4 stripes of the zebra pattern. Note in addition that the bursts with zebra pattern in the meter and decimeter ranges sometimes contain up to several tens of stripes; interpretation of these events using the mechanism considered is difficult and, probably, they have a different origin (Aurass et al. 2003; Zlotnik et al. 2003).

Under reasonable assumptions about the source size the energy density of individual harmonics of Bernstein modes, which is required for generation of a microwave zebra pattern with the observed intensity, is about $10^{-3}-10^{-2} \mathrm{erg} / \mathrm{cm}^{3}$. By assuming that the fraction of energy that corresponds to each harmonic is proportional to the growth rate, the so-called saturated generation regime, we found that the total energy density of all harmonics under these conditions does not exceed $2-3 \%$ of the energy density of accelerated particles. This estimation includes the harmonics with number $s \leq 10$, which are not involved in generation of the observed emission and for which the growth rate reaches $2.5 \times 10^{5} \mathrm{~s}^{-1}$. In the nonsaturated regime of plasma waves generation, the formation of zebra pattern requires additional mechanisms that limit the amplification of harmonics with numbers $s \leq 10$.

The emission generated due to interaction of Bernstein modes has high directivity that decreases the probability of its observation on Earth. However, one can expect that scattering on the small-scale plasma inhomogeneities (with scale about radio emission wave length) during propagation of emission from the source will result both in broadening of the beam angular width and in averaging of the polarization degree. In this case the polarization degree of the observed emission can be estimated as

$\eta=\frac{I_{\mathrm{X}}-I_{\mathrm{O}}}{I_{\mathrm{X}}+I_{\mathrm{O}}}$

where $I_{\mathrm{X}}$ and $I_{\mathrm{O}}$ are the total (integrated over angle) emission intensities of different modes. For the coalescence of harmonics $s^{\prime}=s^{\prime \prime}=11$ (Fig. 9) we obtain $\eta=-90 \%$, for the coalescence of harmonics $s^{\prime}=s^{\prime \prime}=19$ (Fig. 10) $\eta=-73 \%$. The average polarization degree is higher for the coalescence of lower harmonics; also it inreases with a decrease of the angle interval occupied by the Bernstein modes $\left(\Delta \theta^{\prime}, \Delta \theta^{\prime \prime}\right)$. Obviously the emission scattering, besides some decrease in the degree of polarization, will result in a decrease in its intensity.

\section{Conclusion}

This investigation of the processes of generation and nonlinear interaction of Bernstein modes under conditions typical of the microwave zebra pattern source has shown that:

1) This mechanism is able to provide the formation of zebra pattern with equidistant stripes with a frequency interval equal to the electron cyclotron frequency in the source. The interacting waves correspond to the relatively high numbers of cyclotron harmonics $(s>10)$.

2) The generated radio emission has a high polarization degree (up to $100 \%$ ) corresponding to the extraordinary wave.

3) The efficiency of transformation of energy from accelerated electrons into radio emission is high enough to provide the observed emission intensity.

4) The emission is confined within the narrow angle interval around the quasi-transversal propagation direction relative to the magnetic field. In order to increase the probability of detecting emission on Earth the additional process is necessary, which increases the width of the radio beam.

Acknowledgements. This work was supported by the RFBR (grant numbers 02-02-39030, 04-02-39003 and 03-02-16229) and by the Russian Department of Education (grant number E02-3.2-489).

\section{References}

Altyntsev, A. T., Kuznetsov, A. A., Meshalkina, N. S., Rudenko, G. V., \& Yihua Yan 2005, A\&A, 431, 1037

Aurass, H., Klein, K.-L., Zlotnik, E. Ya., \& Zaitsev, V. V. 2003, A\&A, 410, 1001

Chernov, G. P. 1976, Soviet Astron., 20, 582

Chernov, G. P. 1990, Sol. Phys., 130, 75

Dory, R. A., Guest, G. E., \& Harris, E. G. 1965, Phys. Rev. Lett., 14, 131

Kuijpers, J. 1975, A\&A, 40, 405

LaBelle, J., Treumann, R. A., Yoon, P. H., \& Karlicky, M. 2003, ApJ, 593, 1195

Melrose, D. B., \& Sy, W. N. 1972, Aust. J. Phys., 25, 387

Melrose, D. B., \& Dulk, G. A. 1982, ApJ, 259, 844

Mollwo, L., \& Sauer, K. 1977, Sol. Phys., 51, 435

Rosenberg, H. 1972, Sol. Phys., 25, 188

Rudenko, G. V. 2001, Sol. Phys., 198, 5

Stepanov, A. V., Kliem, B., Krüger, A., \& Hildebrandt, J. 1999, ApJ, 524, 961

Trakhtengerts, V. Yu. 1970, Izv. VUZov - Radiofizika, 13, 884

Willes, A. J., \& Robinson, P. A. 1996, ApJ, 467, 465

Willes, A. J., \& Melrose, D. B. 1997, Sol. Phys., 171, 393

Willes, A. J. 1999, Sol. Phys., 186, 319

Zheleznyakov, V. V., \& Zlotnik, E. Ya. 1975a, Sol Phys., 43, 431

Zheleznyakov, V. V., \& Zlotnik, E. Ya. 1975b, Sol. Phys., 44, 461

Zheleznyakov, V. V. 1997, Radioemission in the astrophysical plasma, Moscow, "Yanus-K"

Zlotnik, E. Ya. 1981, A\&A, 101, 250

Zlotnik, E. Ya., Zaitsev, V. V., Aurass, H., Mann, G., \& Hofmann, A. 2003, A\&A, 410, 1011 
A. A. Kuznetsov: Generation of microwave zebra pattern, Online Material p 1

\section{Online Material}




\section{Appendix A: Calculation of the plasma waves growth rate}

Let us consider calculation of the $l$ th term in expression (4). The resonance condition

$\psi=\Gamma\left(\omega-k_{\|} v_{\|}\right)-l \omega_{\mathrm{B}}=0$

in the space of electron velocities represents the ellipse equation with the center at the point $\left\{v_{\|}=v_{0} ; v_{\perp}=0\right\}$ and the semiaxes $a$ and $b$ (Melrose \& Dulk 1982):

$$
\begin{aligned}
a^{2} & =\frac{l^{2} \omega_{\mathrm{B}}^{2} c^{2}\left(k_{\|}^{2} c^{2}+l^{2} \omega_{\mathrm{B}}^{2}-\omega^{2}\right)}{\left(k_{\|}^{2} c^{2}+l^{2} \omega_{\mathrm{B}}^{2}\right)^{2}}, \\
b^{2} & =\frac{c^{2}\left(k_{\|}^{2} c^{2}+l^{2} \omega_{\mathrm{B}}^{2}-\omega^{2}\right)}{k_{\|}^{2} c^{2}+l^{2} \omega_{\mathrm{B}}^{2}} \\
v_{0} & =\frac{\omega k_{\|} c^{2}}{k_{\|}^{2} c^{2}+l^{2} \omega_{\mathrm{B}}^{2}}
\end{aligned}
$$

The integral over $\mathrm{d}^{3} \boldsymbol{p}$ is calculated using conversion to the polar coordinate system $(\rho ; \phi)$ in the velocity space with the center at point $\left\{v_{0} ; 0\right\}$; then, using the properties of delta-function, we come to the integral over one coordinate (the polar angle $\phi$ ) along the arc of the resonance ellipse:

$$
\begin{aligned}
&\left(\operatorname{Im} \varepsilon_{\|}\right)_{l}=-2 \pi^{2} m_{\mathrm{e}}^{4} a b^{2} \frac{\omega_{\mathrm{p}}^{2}}{k^{2}} \int_{0}^{\phi_{\max }}\left[J_{l}^{2}\left(\frac{\Gamma k_{\perp} v_{\perp}}{\omega_{\mathrm{B}}}\right)\right. \\
&\left.\times\left(k_{\|} \frac{\partial f}{\partial p_{\|}}+\frac{l \omega_{\mathrm{B}}}{\Gamma v_{\perp}} \frac{\partial f}{\partial p_{\perp}}\right) \frac{\Gamma^{5} \sin \phi \mathrm{d} \phi}{|\partial \psi / \partial \rho|}\right]\left.\right|_{\psi=0}, \\
& \frac{\partial \psi}{\partial \rho}=\Gamma\left\{\frac{\Gamma l \omega_{\mathrm{B}}}{c^{2}}\left[v_{\|}\left(v_{\|}-v_{0}\right)+v_{\perp}^{2}\right]-k_{\|}\left(v_{\|}-v_{0}\right)\right\} .
\end{aligned}
$$

The integration limits in expression (A.3) correspond to the normal Doppler effect $(l>0)$ and the situation $k_{\|}>0$; in this case

$$
\phi_{\max }= \begin{cases}\pi, & k_{\|}^{2} c^{2} \leq \omega^{2}, \\ \arccos \frac{k_{\|} v_{0}-\omega}{k_{\|} a}, & k_{\|}^{2} c^{2}>\omega^{2} .\end{cases}
$$

The substitution $\psi=0$ means that the components of electron velocity along the resonance curve are calculated by the formulae:

$v_{\|}=v_{0}-a \cos \phi, \quad v_{\perp}=b \sin \phi$.

It was noted above that to calculate the parameter $\operatorname{Im} \varepsilon_{\|}$one has to sum the contributions of different resonance curves (A.3). The parameter $\partial \varepsilon_{\|} / \partial \omega$ that is contained in the formula for the growth rate is determined by the expression (B.12).

\section{Appendix B: Dispersion parameters of interacting waves}

The polarization vectors of waves in plasma satisfy the following conditions:

$$
\left[k^{2} \delta_{i j}-k_{i} k_{j}-\frac{\omega^{2}}{c^{2}} \varepsilon_{i j}(\omega, \boldsymbol{k})\right] a_{j}(\boldsymbol{k})=0,
$$

$\left(\boldsymbol{a} a^{*}\right)=1$,

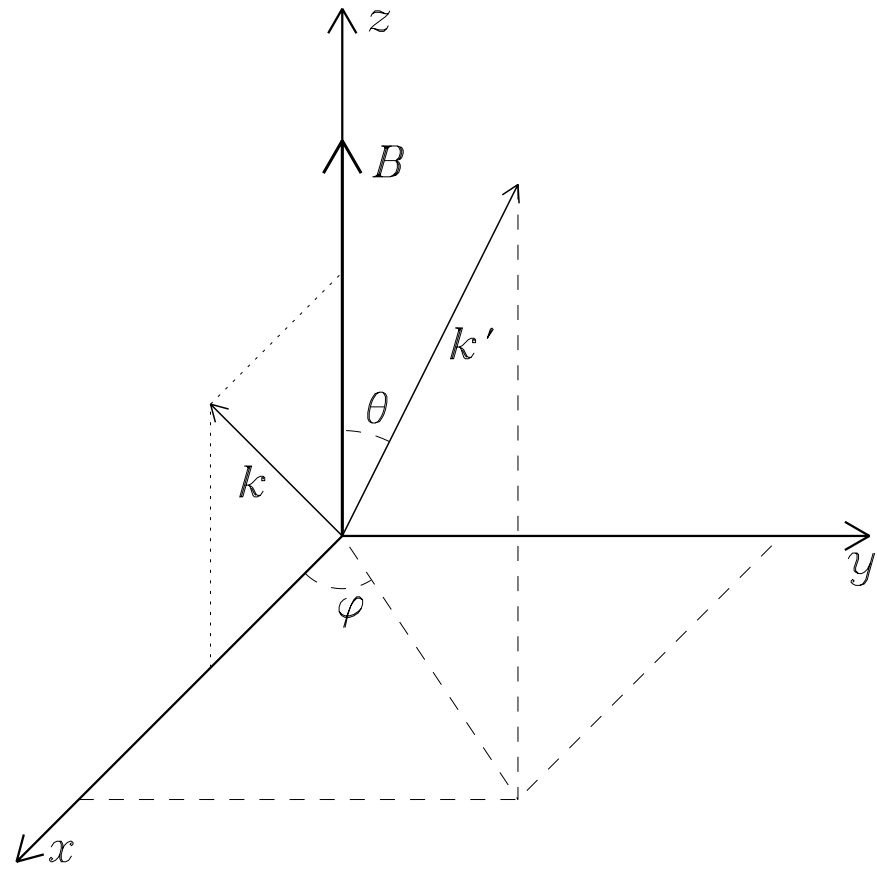

Fig. B.1. The coordinate system used in this paper.

where $\varepsilon_{i j}, \omega$, and $\boldsymbol{k}$ are the tensor of dielectric permeability, frequency, and wave vector for the considered wave type, respectively.

The parameter $R(\boldsymbol{k})$ is determined by (Melrose \& Sy 1972; Willes \& Robinson 1996)

$$
\begin{aligned}
& R(\boldsymbol{k})=\left|\frac{\omega}{\partial\left(\omega^{2} \epsilon\right) / \partial \omega}\right| \\
& \epsilon=a_{i}^{*}(\boldsymbol{k}) \varepsilon_{i j}(\omega, \boldsymbol{k}) a_{j}(\boldsymbol{k}) .
\end{aligned}
$$

\section{B.1. Electromagnetic waves}

To describe the dispersion properties of electromagnetic waves we use the cold plasma approximation, that is, the thermal motion of particles is neglected, $T_{0} \rightarrow 0$ (Melrose \& Sy 1972; Willes \& Melrose 1997; Zheleznyakov 1997). In this case the refraction index $N=k c / \omega$ is

$$
\begin{aligned}
& N_{\sigma}^{2}=1-\frac{2 v(1-v)}{2(1-v)-u \sin ^{2} \theta+\sigma \sqrt{D}} \\
& D=u^{2} \sin ^{4} \theta+4 u(1-v)^{2} \cos ^{2} \theta
\end{aligned}
$$

where $\theta$ is the angle between the wave vector and the magnetic field, $u=\omega_{\mathrm{B}}^{2} / \omega^{2}, v=\omega_{\mathrm{p}}^{2} / \omega^{2}, \sigma=-1$ for the extraordinary wave, $\sigma=+1$ for the ordinary wave.

We consider the coordinate system (Fig. B.1) where (i) $z$ axis is directed along the magnetic field; (ii) $x$ axis is perpendicular to the magnetic field and lies in the same plane with the magnetic field vector $\boldsymbol{B}$ and the wave vector of the electromagnetic wave $\boldsymbol{k}$; and (iii) $y$ axis is perpendicular to the vectors $\boldsymbol{B}$ and $\boldsymbol{k}$. In this system the wave vector of the 


\section{A. A. Kuznetsov: Generation of microwave zebra pattern, Online Material p 3}

electromagnetic wave takes the form $\boldsymbol{k}=\{k \sin \theta ; 0 ; k \cos \theta\}$, and the polarization vector of the electromagnetic wave equals

$\boldsymbol{a}=\frac{\left\{T_{\sigma} \cos \theta+L_{\sigma} \sin \theta ; i ;-T_{\sigma} \sin \theta+L_{\sigma} \cos \theta\right\}}{\sqrt{L_{\sigma}^{2}+T_{\sigma}^{2}+1}}$,

$T_{\sigma}=\frac{2 \sqrt{u}(1-v) \cos \theta}{u \sin ^{2} \theta-\sigma \sqrt{D}}$,

$L_{\sigma}=\frac{v \sqrt{u} \sin \theta+T_{\sigma} u v \sin \theta \cos \theta}{1-u-v+u v \cos ^{2} \theta}$.

The parameter $\partial\left(\omega^{2} \epsilon\right) / \partial \omega$ for the electromagnetic waves is

$$
\begin{aligned}
\frac{\partial\left(\omega^{2} \epsilon\right)}{\partial \omega}=2 \omega N_{\sigma} & \frac{\partial\left(\omega N_{\sigma}\right)}{\partial \omega} \frac{T_{\sigma}^{2}+1}{L_{\sigma}^{2}+T_{\sigma}^{2}+1}, \\
N_{\sigma} \frac{\partial\left(\omega N_{\sigma}\right)}{\partial \omega}= & 1+\frac{v \sqrt{u} T_{\sigma} \cos \theta}{2\left(T_{\sigma}-\sqrt{u} \cos \theta\right)^{2}} \\
& \times\left[1+\frac{(1+v)\left(1-T_{\sigma}^{2}\right)}{(1-v)\left(1+T_{\sigma}^{2}\right)}\right] .
\end{aligned}
$$

In the weakly anisotropic plasma $\left(\omega_{\mathrm{p}} \gg \omega_{\mathrm{B}}\right)$ the ratio of electric to total energy for the electromagnetic waves $R \simeq 1 / 2$ for both modes.

\section{B.2. Bernstein modes}

It was said above that the used in this paper coordinate system is connected with the direction of the wave vector of the electromagnetic wave. The wave vector of plasma waves is determined by three parameters $k, \theta$, and $\varphi$ (Fig. B.1). Let us consider first the case where $\varphi=0$ : here the wave vector of the Bernstein modes takes the form $\boldsymbol{k}=\{k \sin \theta ; 0 ; k \cos \theta\}$, and the following relations between the components of the polarization vector are satisfied:

$\frac{a_{y}}{a_{x}}=-\frac{\varepsilon_{x y}}{N^{2}-\varepsilon_{y y}}, \quad \frac{a_{z}}{a_{x}}=\frac{N^{2} \sin \theta \cos \theta}{N^{2} \sin ^{2} \theta-\varepsilon_{z z}}$.

The tensor of dielectric permeability $\varepsilon_{i j}$ for the Maxwellian plasma with nonzero temperature is given, for example, in the book by Zheleznyakov (1997). For the quasi-transversal propagation $(\cos \theta \ll 1)$ one obtains the polarization vector of the Bernstein modes in the form:

$\boldsymbol{a} \simeq\left\{1,-\frac{i v \sqrt{u} \xi}{N^{2}-2 u v \lambda \xi}, \frac{N^{2} \cos \theta}{N^{2}-1+v+u \lambda}\right\}$,

$\xi=-2 \omega^{2} \mathrm{e}^{-\lambda} \sum_{r=1}^{\infty} \frac{r^{2}\left[I_{r}(\lambda)-I_{r}^{\prime}(\lambda)\right]}{\omega^{2}-r^{2} \omega_{\mathrm{B}}^{2}}$.

Here the designations are similar to the previous paragraph: $N=k c / \omega, u=\omega_{\mathrm{B}}^{2} / \omega^{2}, v=\omega_{\mathrm{p}}^{2} / \omega^{2}$. When obtaining the polarization vector, we used the property that the Bernstein modes are almost longitudinal waves $\left(\left|a_{x}\right| \simeq 1,\left|a_{y, z}\right| \ll 1\right)$. In addition, the condition $\varepsilon_{x x} \simeq \varepsilon_{\|}=0$ that follows from the dispersion Eq. (1) allows us to simplify the expressions for the components $\varepsilon_{y y}$ and $\varepsilon_{z z}$ of the dielectric permeability tensor.
If the azimuthal angle $\varphi$ is nonzero, then the abovementioned wave vector and polarization vector should be multiplied by the rotation matrix:

$M(\varphi)=\left(\begin{array}{ccc}\cos \varphi & -\sin \varphi & 0 \\ \sin \varphi & \cos \varphi & 0 \\ 0 & 0 & 1\end{array}\right)$

The other dispersion parameters of the Bernstein modes used in this paper, have the form:

$\frac{\partial \varepsilon_{\|}}{\partial \omega}=4 \omega \omega_{\mathrm{p}}^{2} \frac{\mathrm{e}^{-\lambda}}{\lambda} \sum_{r=1}^{\infty} \frac{r^{2} I_{r}(\lambda)}{\left(\omega^{2}-r^{2} \omega_{\mathrm{B}}^{2}\right)^{2}}$,

$\frac{\partial\left(\omega^{2} \epsilon\right)}{\partial \omega} \simeq \omega^{2} \frac{\partial \varepsilon_{\|}}{\partial \omega}$

The group velocity is found using the rule of differentiation of the implicit function $\varepsilon_{\|}=0$ :

$\frac{\partial \omega}{\partial k_{\perp}}=-\frac{\partial \varepsilon_{\|} / \partial k_{\perp}}{\partial \varepsilon_{\|} / \partial \omega}, \quad \frac{\partial \varepsilon_{\|}}{\partial k_{\perp}}=\frac{2}{k_{\perp}}(1-v \xi)$.

Under the conditions considered in this paper the ratio of electric to total energy for the Bernstein modes is $R \simeq 10^{-2}$.

\section{Appendix C: Calculation of the efficiency of nonlinear interaction}

The tensor of plasma nonlinear conductivity used in this paper has the following form (Trakhtengerts 1970):

$$
\begin{aligned}
& \mathrm{S}_{i n m}\left(\boldsymbol{k}, \boldsymbol{k}^{\prime}, \boldsymbol{k}^{\prime \prime}\right)=\frac{1}{e n_{0}}\left[\frac{k_{l}^{\prime \prime}}{\omega^{\prime \prime}} \sigma_{i n}^{\prime} \sigma_{l m}^{\prime \prime}\right. \\
& \left.-\frac{4 \pi i}{\omega_{\mathrm{p}}^{2}} \sigma_{i j} \sigma_{l n}^{\prime} \sigma_{j m}^{\prime \prime} k_{l}^{\prime \prime}+\frac{k_{j}^{\prime \prime}}{\omega^{\prime \prime}}\left(\sigma_{i j} \sigma_{m n}^{\prime}-\sigma_{i m} \sigma_{j n}^{\prime}\right)\right],
\end{aligned}
$$

where $e$ is the electron charge. The tensor of linear conductivity $\sigma_{i j}(\omega, \boldsymbol{k})$ for each of the interacting waves is

$\sigma_{i j}(\omega, \boldsymbol{k})=\frac{\omega}{4 \pi i}\left[\varepsilon_{i j}(\omega, \boldsymbol{k})-\delta_{i j}\right]$,

where the corresponding frequency and dielectric permeability tensor should be substituted.

By using the relations (B.1), the convolution $S$ (15) can be transformed to the form:

$$
\begin{aligned}
S= & \frac{\left(\boldsymbol{\tau}^{\prime} \boldsymbol{a}^{*}\right)\left(\boldsymbol{\tau}^{\prime \prime} \boldsymbol{k}^{\prime \prime}\right)+\left(\boldsymbol{\tau}^{*} \boldsymbol{k}^{\prime \prime}\right)\left(\boldsymbol{\tau}^{\prime} \boldsymbol{a}^{\prime \prime}\right)-\left(\boldsymbol{\tau}^{*} \boldsymbol{a}^{\prime \prime}\right)\left(\boldsymbol{\tau}^{\prime} \boldsymbol{k}^{\prime \prime}\right)}{\omega^{\prime \prime}} \\
& +\frac{\left(\boldsymbol{\tau}^{\prime \prime} \boldsymbol{a}^{*}\right)\left(\boldsymbol{\tau}^{\prime} \boldsymbol{k}^{\prime}\right)+\left(\boldsymbol{\tau}^{*} \boldsymbol{k}^{\prime}\right)\left(\boldsymbol{\tau}^{\prime \prime} \boldsymbol{a}^{\prime}\right)-\left(\boldsymbol{\tau}^{*} \boldsymbol{a}^{\prime}\right)\left(\boldsymbol{\tau}^{\prime \prime} \boldsymbol{k}^{\prime}\right)}{\omega^{\prime}} \\
& \left.-\frac{4 \pi i}{\omega_{\mathrm{p}}^{2}}\left[\left(\boldsymbol{\tau}^{*} \boldsymbol{\tau}^{\prime \prime}\right)\left(\boldsymbol{\tau}^{\prime} \boldsymbol{k}^{\prime \prime}\right)+\left(\boldsymbol{\tau}^{*} \boldsymbol{\tau}^{\prime}\right)\left(\boldsymbol{\tau}^{\prime \prime} \boldsymbol{k}^{\prime}\right)\right]\right\} /\left(e n_{0}\right) .
\end{aligned}
$$

Here vectors $\tau_{i}^{\prime}\left(\boldsymbol{k}^{\prime}\right)=\sigma_{i j}^{\prime} a_{j}^{\prime}, \tau_{j}^{*}(\boldsymbol{k})=\sigma_{i j} a_{i}^{*}$ are defined as

$$
\begin{aligned}
& \tau_{j}^{*}=\frac{c^{2}}{4 \pi i \omega}\left[\left(k^{2}-\frac{\omega^{2}}{c^{2}}\right) a_{j}^{*}-\left(\boldsymbol{k} \boldsymbol{a}^{*}\right) k_{j}\right], \\
& \tau_{i}^{\prime}=\frac{c^{2}}{4 \pi i \omega^{\prime}}\left\{\left[\left(k^{\prime}\right)^{2}-\frac{\left(\omega^{\prime}\right)^{2}}{c^{2}}\right] a_{i}^{\prime}-\left(\boldsymbol{k}^{\prime} \boldsymbol{a}^{\prime}\right) k_{i}^{\prime}\right\} .
\end{aligned}
$$


The vector $\tau_{i}^{\prime \prime}\left(\boldsymbol{k}^{\prime \prime}\right)=\sigma_{i j}^{\prime \prime} a_{j}^{\prime \prime}$ is calculated in the same way as $\tau_{i}^{\prime}$.

By using the properties of delta-function the integral (13)

can be reduced to the integral over the direction angles of the wave vector $\boldsymbol{k}^{\prime}$ :

$$
\begin{aligned}
\frac{\mathrm{d} W_{\boldsymbol{k}}(\boldsymbol{k})}{\mathrm{d} t}= & \iint\left\{\left[\Pi\left(\boldsymbol{k}, \boldsymbol{k}^{\prime}, \boldsymbol{k}^{\prime \prime}\right) W_{\boldsymbol{k}^{\prime}}^{\prime}\left(\boldsymbol{k}^{\prime}\right) W_{\boldsymbol{k}^{\prime \prime}}^{\prime \prime}\left(\boldsymbol{k}^{\prime \prime}\right)\right.\right. \\
& \left.\times \frac{k^{\prime 2} \sin \theta^{\prime}}{\left|\partial w / \partial k^{\prime}\right|}||_{w=0, \boldsymbol{k}^{\prime \prime}=\boldsymbol{k}-\boldsymbol{k}^{\prime}}\right\} \mathrm{d} \theta^{\prime} \mathrm{d} \varphi^{\prime},
\end{aligned}
$$

$w=\omega(\boldsymbol{k})-\omega^{\prime}\left(\boldsymbol{k}^{\prime}\right)-\omega^{\prime \prime}\left(\boldsymbol{k}-\boldsymbol{k}^{\prime}\right)$.

The double integral (C.5) is calculated numerically (Willes \& Melrose 1997; Willes 1999); for each pair of values $\left(\theta^{\prime}, \varphi^{\prime}\right)$ the absolute value of the wave vector $k^{\prime}$ is found by the numerical solution of the equation $w\left(\boldsymbol{k}^{\prime}\right)=0$ in the interval $k_{\min }^{\prime} \leq k^{\prime} \leq k_{\max }^{\prime}$ (the parameters of the electromagnetic wave $\omega$ and $\boldsymbol{k}$ are assumed to be given in this step), then the vector $\boldsymbol{k}^{\prime \prime}$ is found as $\boldsymbol{k}^{\prime \prime}=\boldsymbol{k}-\boldsymbol{k}^{\prime}$. The derivative of the frequency difference which is contained in the expression (C.5) equals

$\frac{\partial w}{\partial k^{\prime}}=-\frac{\partial \omega^{\prime}}{\partial k^{\prime}}+\left.\left[\frac{\left(\boldsymbol{k}^{\prime} \boldsymbol{k}^{\prime \prime}\right)}{k^{\prime} k^{\prime \prime}} \frac{\partial \omega^{\prime \prime}}{\partial k^{\prime \prime}}\right]\right|_{\boldsymbol{k}^{\prime \prime}=k-\boldsymbol{k}^{\prime}}$.

When calculating the derivatives of the frequency on the wave vector we can assume that $k^{\prime} \simeq k_{\perp}^{\prime}, k^{\prime \prime} \simeq k_{\perp}^{\prime \prime}$, and use the expression (B.14). 